\title{
A Review on Buckling Analysis of Functionally Graded Plates Under Thermo-Mechanical Loads
}

\author{
Ahmed Hassan Ahmed Hassan ${ }^{a *}$, Naci Kurgan ${ }^{b}$ \\ ${ }^{a, b}$ Ondokuz, Mayis University, Faculty of Engineering, Department of Mechanical Engineering, Samsun, Turkey. \\ E-mail address: 15210457@stu.omu.edu.tr ${ }^{\mathrm{a}^{*}}$, naci.kurgan@ omu.edu.tr ${ }^{\mathrm{b}}$
}

ORCID numbers of authors

0000-0002-4880-0184 a, 0000-0001-7297-7249

Received date: 18.04 .2019

Accepted date: 21.05 .2019

\begin{abstract}
Functionally graded materials (FGM) are increasingly used in the engineering field. In many applications, FGMs are modelled as plates. Plates made of functionally graded materials (FGPS) are mostly designed to perform under elevated temperatures. In those circumstances, they are often under the combined effect of thermal and mechanical loads. There have been many studies on buckling analysis of FGP under either mechanical or thermal loads; however, only a few studies have addressed the combined effect of both loads acting together. This article focuses on the review of research on buckling analysis of FGP under the combined thermal and mechanical loads.
\end{abstract}

Keywords: Functionally graded material; plate; FGP models; thermo-mechanical buckling; solution methods.

\section{Introduction}

Functionally graded materials (FGMs) are advanced inhomogeneous composite materials in which graded interlayer separates different materials of the composite structure. FGM's concept is to replace the sudden change in composition that occurs at the interface between different materials, with a compositionally graded phase, aiming at reducing stress concentrations through the structure. This microstructure variation in FGM occurs with a specific function through one or more dimensions of the volume. The concept of gradual variation in material is found in nature. Many organic structures have gradual variation through one or more dimensions. For example, human bones and bamboo trees. More examples are available in [1]. FGM's concept was first explored theoretically early in the seventies of the previous century by Shen and Bever [2, 3]. More than ten years later, in Japan, 1984, FGM concept was first implemented in engineering application. That first implementation was in design of thermalresistant structure. Since then there have been intensive research and various implementations of FGM concept in various engineering applications. Recently, FGM has been introduced to gear structures [4, 5], turning tools [6], drilling wicks [7] and many other applications including tubes, rotating disks and sport instruments [8]. In addition, FGMs have found applications as 
electrical field grading insulators [9]. Other important applications of FGMs are in the medical field. FGMs have been intensively investigated as biomaterials [10,11], especially in dentistry as dental implants and crowns [12,13], bone plates [14], bone implants [15] and knee joints [16].

In literature, there are various methods of modeling, analysis, fabrication and characterizing of FGM parts available. Relatively thin structures with a wide flat planar surface are normally called plates. When the concept of FGM is implemented to a plate then it is called functionally graded plate (FGP). FGPs are generally designed to withstand high thermal loads and it often experiences some additional mechanical loads. Therefore, FGPs often have to be designed to withstand the combined effect of the thermal and mechanical loads. FGPs provide designers with the ability to tailor material response so desired performance can be achieved. FGPs found wide range of applications especially space vehicles where it was first implemented [1]. Other fields also have started to adopt and benefit from this relatively new concept. As an example, Cooley [17] designed an exhaust wash structure as FGP to be used in aircrafts those have internally exhausted engines. There have been many studies on analysis of FGP under either mechanical or thermal loads; however, only a few studies have addressed the combined effect of both loads acting together. One main analysis usually conducted in plate design is buckling analysis. When a flat plate experiences compressive in-plane loads, resulting from compression loads at its edges or thermal loads with constrained edges, buckling and post-buckling analysis have to be conducted to ensure correct prediction of the plate's behavior. Review articles those cover FGP buckling under combined thermal and mechanical loads are rare. Swaminathan [18] reviewed thermo-mechanical buckling analysis of FGPs briefly in a subsection of a wide review that also contains stress and vibration analysis. Later on, same author, Swaminathan [19] reviewed modeling and solution methods used in thermal buckling of FGPs, but did not separate buckling under combined thermal and mechanical loads from thermal buckling studies. It is obvious that the combined loading is the general case. Therefore, focusing on it and showing which FGPs' models, shapes, configurations and solution methods are used is a step towards filling gaps in this study field.

In this review article, FGP buckling under combined thermal and mechanical loads studies are reviewed aiming at providing a closer look at this specific analysis and pointing at existing gaps in its literature. This review starts with brief on FGP definition as a plate and as functionally graded material and about their geometries and configurations, those appear in literature. Then, in the third section, the implemented models are listed with brief description. Followed by the fourth section briefing methods to characterize FGP properties. In the fifth section, buckling, thermal buckling and thermo-mechanical buckling are briefly discussed. Solution methods are reviewed in the sixth section. Suggestions and comments are included in the last section.

\section{FGP definition, shapes and configuration}

FGP is a plate that has the concept of FGM implemented to its composition. Reddy [20] defined plate as: "A plate is a structural element with planform dimensions that are large compared to its thickness and is subjected to loads that cause bending deformation in addition to stretching". A rectangular plate is shown in Fig. 1 with typical coordinate system and names of characteristic dimensions. Normally, the length of the longer inplane edge of the plate is referred to as (a), where the shorter is referred to as (b), while the out-of-plane depth, a.k.a. thickness, is referred to as (h). 


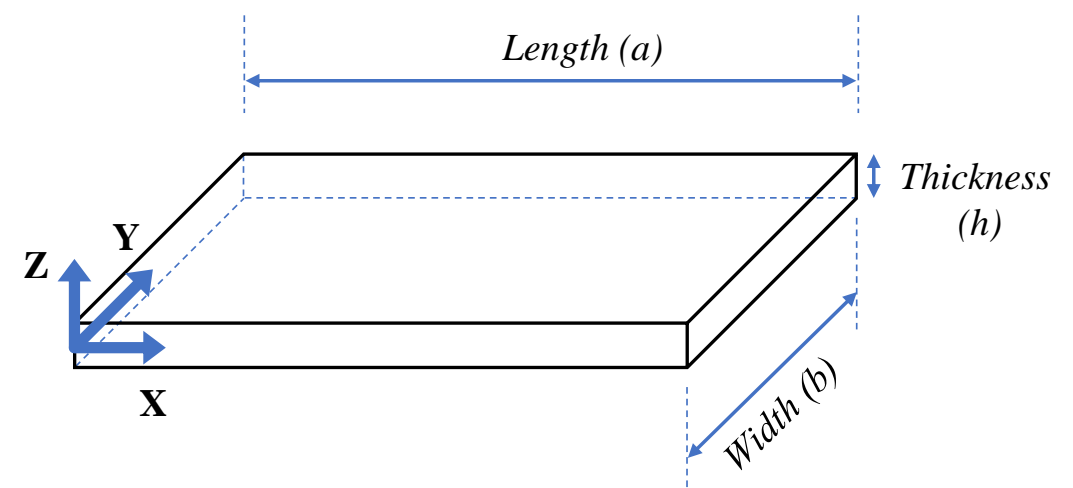

Fig. 1. Rectangular plate structure

In literature, various shapes and configurations are considered in thermo-mechanical buckling analysis of FGPs. Different shapes and configurations come from the many aspects those define a FGP as a plate and those define FGP as FGM.

\subsection{FGP's plate characteristics}

The first aspect that defines FGP as a plate is its thickness to side ratio. Plates may be classified according to the ratio of a its typical inplane dimension to its thickness [21] into thick, moderately thick and thin plates. All the three classes have been found in literature of thermomechanical buckling analysis of FGPs. Few examples for studies that considered thin plates are [22] and [23]; moderately thick plates [24] and [25]; and thick plates [26] and [27]. Another aspect is the thickness variation; in the literature under focus, only FGPs with constant thickness were found. Next aspect for defining a plate is the scale of its dimensions. According to this aspect, FGP can be classified into nano-plates, micro- plates and "normal" plates. Nano-FGP and micro-FGPs have been studied for thermo-mechanical buckling by many researchers, for example [28]. Nano-FGP and micro-FGP are out of the scope of this review article.

Next aspect is the shape of the FGP. The rectangular is the most studied shape of FGP for the thermo-mechanical buckling analysis. However, other FGP shapes have been also studied. Other considered shapes are the annular FGP analyzed numerically by Shariyat [29], skew FGP analyzed numerically by Taj [30] and Yu [31], square FGP analyzed by Kowal-Michalska [22] using approximate analytical method, and circular FGP studied by Kiani [32], Fallah [33] and Li [34]. Various special configurations have been considered in literature. Stiffened FGPs have been studied by Cong [25], Van [35] and Duc [27]. FGP with holes and perforated FGP have been considered by Lal [36] and Shariyat [29], respectively. Porous FGP have been analyzed by Cong [37] using approximate analytical methods. Cracked FGP have been analyzed by Fan [38] using approximate analytical methods.

\subsection{FGP resting on elastic foundation}

An important configuration is FGP resting on elastic foundation. The simplest model for the elastic foundation is the Winkler model, which regards the foundation as a series of separated springs without coupling effects between each other. Pasternak model adds a shear layer to the Winkler model as a parameter. Pasternak model is widely used to describe the mechanical behavior of structure-foundation interactions [39]. More complicated foundation model is Kerr's model, which adds a third parameter as an additional shear layer [40]. 
In the literature of thermo-mechanical buckling analysis of FGP, the only used foundation model is the Pasternak model. FGP on Pasternak foundation has been analyzed using approximate analytical methods by Cong [25, 37], Bakora [26], Duc [27, 41], Bateni [42], Chikh [43], Yu [44], Fan [38] and Shen [45, 46]. Whereas Mansouri [47] and Shams [48] used numerical methods. It is worth mentioning that in all these studies only the rectangular plate was considered.

\subsection{Sandwich FGP}

Lastly, we have sandwich FGP configuration. FGP may be included in sandwich structure as core material or as face sheet material as shown in Fig. 2. In literature of thermo-mechanical buckling analysis, both structures have been considered and studied. Tung [24], Yu [44], Shen [45, 46, 49] and Yaghoobi [50] studied various FGP sandwich configurations. It is noticed that all these studies implemented approximate analytical methods. In addition, all of these studies considered only the rectangular FGP.

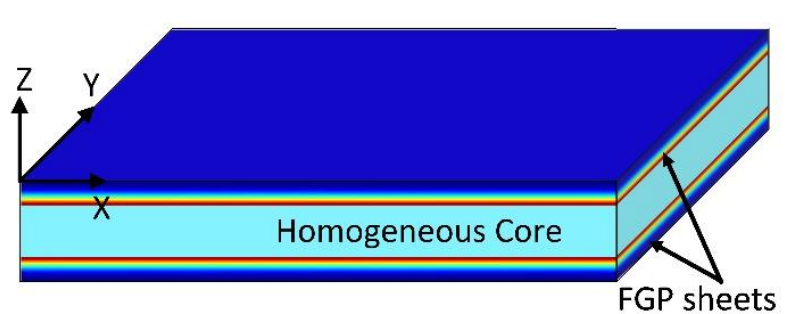

a) FGM sheets on homogenous core

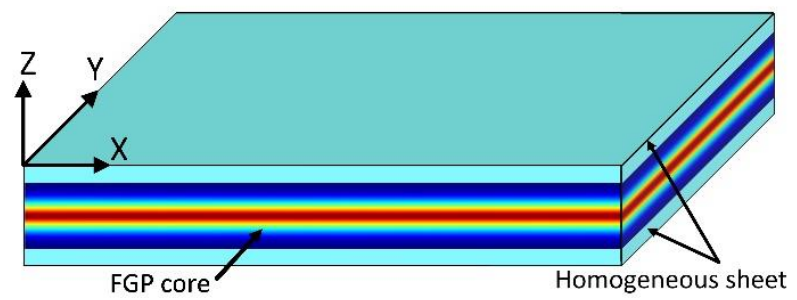

b) Homogenous sheets on FGM core

Fig. 2. Sandwich FGP structures

\subsection{FGP's FGM characteristics}

The second part of aspects define FGP as FGM. The first aspect in this part is the dimensionality of FGP, which refers to the number of dimensions through which properties variation occurs. Composition variation happens through the thickness, or the in-plane two dimensions $[51,52]$ as shown in Fig. 3. In literature of thermo-mechanical buckling analysis of FGP, the only studied FGP is the 1-D that has its variation through the thickness.

The Next aspect is the symmetry of variation of FGP. Generally, in thermal buckling analysis, the plate symmetry is important in determining if there will be a bifurcation point or not. Fig. 4 shows a symmetric and non-symmetric rectangular FGPs. Lack of symmetry is considered as serious initial imperfection in the plate, that prevent reaching a bifurcation point except for specific loading configurations and boundary conditions. Examples for studies considered symmetric FGP: [24, 43, 49, 50, 53, 54]; and for non-symmetric FGPs: [22, 38, 42, 44].

Another important aspect is the continuity of the variation in FGP. FGP may be studied as single layer with continuous variation with a function determines its properties at every point of the plate, or as laminated homogenous layers with specific thickness of each, as shown in Fig. 5. Usage of one of these models depends on the solution methods. 


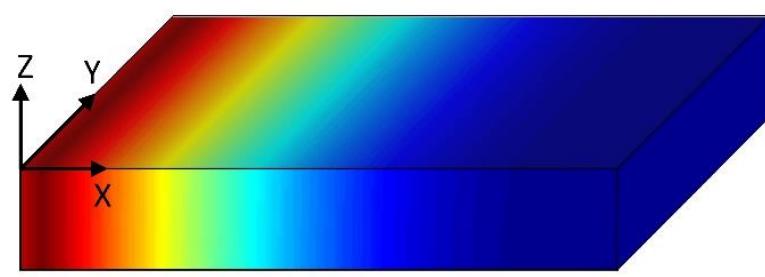

a) Inplane one-dimensional FGP

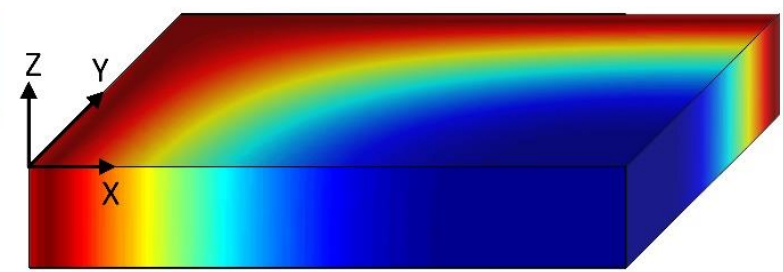

b) Inplane two-dimensional FGP

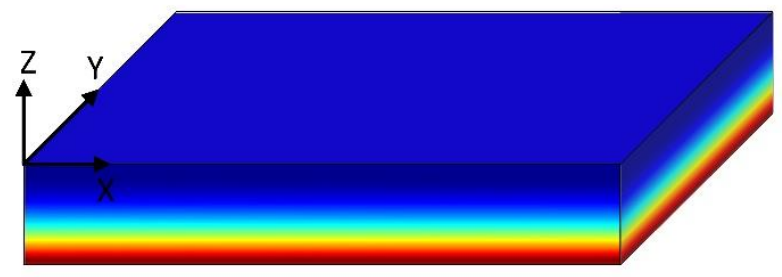

c) Out-of-plane one-dimensional FGP

Fig. 3. FGP's property/composition variation dimensionality

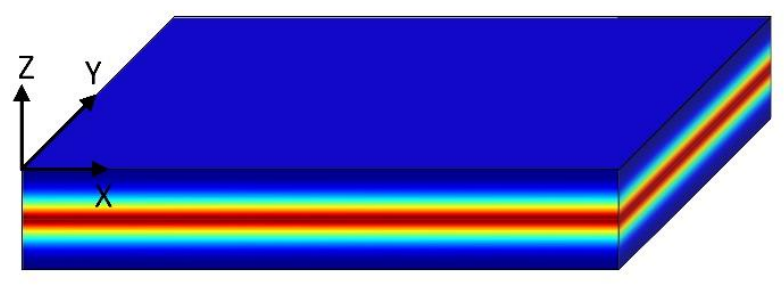

a) Symmetric FGP

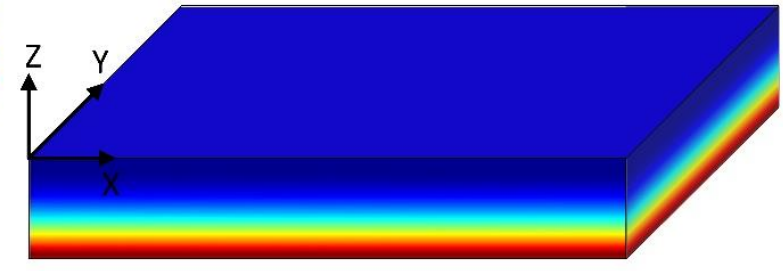

b) Non-symmetric FGP

Fig. 4. Symmetry of FGP

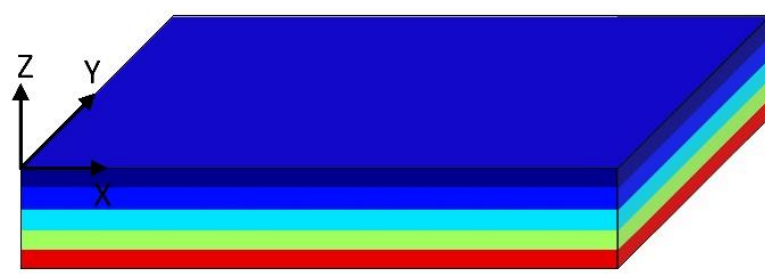

a) Stepped (laminated) FGP

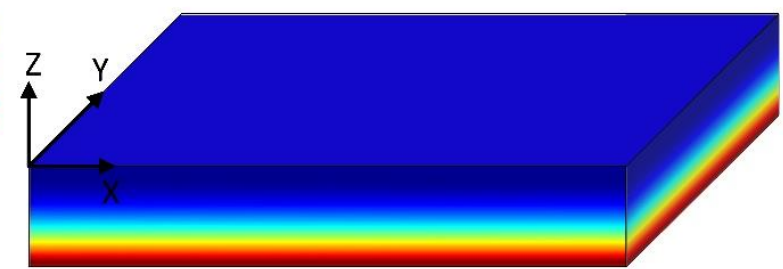

b) Continuous FGP

Fig. 5. Variation continuity of FGP 


\section{FGP models}

Modelling and Analysis of FGPs' behavior are discussed in many books of plate mechanics, e.g. [20, 21, 55-62]. Thermo-mechanical buckling of FGPs has been studied mostly using various models based on plate theories. However, other models based on theory of elasticity are rarely used for this analysis. Following is a brief on the models used in thermo-mechanical buckling of FGPs.

\subsection{Models based on elasticity theory}

Theory of elasticity is based on the concepts of equilibrium, continuum and a material constitutive relationship to analyze any structure [58]. While FGPs can be analyzed using elasticity theory providing high accuracy solutions, it requires more solving effort in both analytical and numerical solution methods. Using elasticity theory to analyze plates provide benchmark to assess accuracy and validity of other simplified methods [63]. In literature of thermo-mechanical buckling analysis of FGP, an elasticity theory-based model has been used just once in the recent study of Shariyat [29].

\subsection{Models based on plate theories}

Plate theories are based on mechanics-of-material approach. Mechanics-of-material approach is based on assuming suitable hypothesis regarding the geometry of deformation, which simplify the problem resulting in simpler models [20]. Models based on plate theories contain equivalent single layer (ESL) models and laminated layer models. In literature of FGP thermomechanical buckling, laminated layer model has been used just once by Kowal-Michalska [22]. Equivalent Single Layer theories (ESL) are based on the assumption that properties, and hence displacement, through the thickness of the plate can be expressed by a function. This function may be polynomial or non-polynomial. Because of the smallness of the plate thickness, it is suitable to analysis the plate as two-dimensional structure with assumed relations describe variation of deformation and stresses through the thickness. comprehensive view of ESL is presented at [64]. ESL-based models those used in thermo-mechanical buckling analysis of FGPs are briefly described as follows.

\subsubsection{Classical plate theory (CPT)}

This theory was developed in 1888 by Love using assumptions proposed by Kirchhoff. It is also called "Kirchhoff plate theory", "Kirchhoff-Love plate theory" or "Thin plate theory". It is an extension of "Euler-Bernoulli beam theory". CPT ignores both shear and normal deformation effects. Assumptions of this theory are expressed as restrictions of transverse normals, i.e. straight lines normal to the mid-surface before deformation. CPT assumes that transverse normals remain straight, normal to the neutral plane and at the same length after deformation [20]. Displacement field of the CPT can be written as shown in Eq. (1), in which $(u, v, w)$ are the displacements of any point $(x, y, z)$ of the plate in $\mathrm{X}, \mathrm{Y}$ and Z-directions respectively; $\left(u_{0}, v_{0}, w_{0}\right)$ are the displacements of point $(x, y)$ at the neutral plane in $\mathrm{X}, \mathrm{Y}$ and Z-directions respectively. CPT models are implemented in FGP thermo-mechanical buckling analysis by Amoushahi [65] for rectangular FGP. In addition to buckling, post-buckling has been studied using CPT model by Tung [66] for rectangular FGP and Li [34] for circular FGP. 


$$
\begin{gathered}
u(x, y, z)=u_{0}(x, y)-z \frac{\partial w_{0}}{\partial x} \\
v(x, y, z)=v_{0}(x, y)-z \frac{\partial w_{0}}{\partial y} \\
w(x, y, z)=w_{0}(x, y)
\end{gathered}
$$

\subsubsection{First order shear deformation theory (FSDT)}

Also called "Reissner-Hencky-Bollé-Mindlin plate Theory", "Reissner-Mindlin plate Theory", "Mindlin plate Theory", "first-order theory" or "Moderately thick plate theory" [58]. It forms an extended version of "Timoshenko beam theory". FSDT extends the CPT by relaxing the normality restriction of the transverse normals to the neutral plane. FSDT assumes that transverse normals remain straight and at the same length after deformation. FSDT yields a constant value of transverse shear strain through the thickness of the plate, and thus requires shear correction factors to account for the parabolic distribution of shear strain [20]. Displacement field of the FSDT can be written as shown in Eq. (2).

$$
\begin{gathered}
u(x, y, z)=u_{0}(x, y)+z \phi_{x}(x, y) \\
v(x, y, z)=v_{0}(x, y)+z \phi_{y}(x, y) \\
w(x, y, z)=w_{0}(x, y)
\end{gathered}
$$

$(u, v, w)$ are the displacements of any point $(x, y, z)$ of the plate in $\mathrm{X}, \mathrm{Y}$ and Z-directions respectively; $\left(u_{0}, v_{0}, w_{0}\right)$ are the displacements of point $(x, y)$ at the neutral plane in $\mathrm{X}$, Y and Z-directions respectively; $\left(\phi_{x}, \phi_{y}\right)$ are the rotations of a transverse normal about the Y- and Xaxes, respectively. FSDT models are implemented in FGP thermo-mechanical buckling analysis in [23-25, 31, 33, 48, 50, 53, 67-72] for rectangular FGPs and in [73] for circular FGP.

\subsubsection{Higher order shear deformation theory (HSDT)}

Also called refined nonlinear theory of plates. In order to eliminate the need for shear correction factor needed for FSDT, higher shear deformation theories were proposed. In addition, HSDT yields more accurate results than FSDT along with more computational efforts. Theoretically, shear deformation order may reach any order to achieve desired degree of accuracy; but in practice, the third order shear deformation is quite enough giving satisfying results with affordable computational effort. There are many third-order shear deformation theories (TSDT), but the most widely used is Reddy's TSDT, proposed by Reddy [74]. Third order shear deformation theories are reviewed in [75]. Displacement field of the TSDT can be written as shown in Eq. (3).

$$
\begin{gathered}
u(x, y, z)=u_{0}(x, y)+z \psi_{x}(x, y)+z^{2} \xi_{x}(x, y)+z^{3} \zeta_{x}(x, y) \\
v(x, y, z)=v_{0}(x, y)+z \psi_{y}(x, y)+z^{2} \xi_{y}(x, y)+z^{3} \zeta_{y}(x, y) \\
w(x, y, z)=w_{0}(x, y)
\end{gathered}
$$


$\left(u_{0}, v_{0}, w_{0}\right)$ denote the displacements of a point $(x, y)$ on the midplane, and $\left(\psi_{x}, \psi_{y}\right)$ are the rotations of normals to midplane about the $\mathrm{Y}$ and $\mathrm{X}$-axes, respectively. TSDT models are implemented in FGP thermo-mechanical buckling analysis in [26, 76-78] for rectangular FGPs and in [30] for skew FGP.

Reddy's TSDT is a TSDT in which the functions $\left(\xi_{x}, \xi_{y}, \zeta_{x}, \zeta_{y}\right)$ are determined using the condition that the transverse shear stresses at the top and bottom surfaces are zero. By finding $\left(\psi_{x}, \psi_{y}, \xi_{x}, \xi_{y}, \zeta_{x}, \zeta_{y}\right)$, Reddy’s TSDT displacement field reduced to Eq. (4).

$$
\begin{gathered}
u(x, y, z)=u_{0}(x, y)+z \phi_{x}(x, y)-\frac{4 z^{3}}{3 h^{2}}\left(\phi_{x}+\frac{\partial \omega_{0}}{\partial x}\right) \\
v(x, y, z)=v_{0}(x, y)+z \phi_{y}(x, y)-\frac{4 z^{3}}{3 h^{2}}\left(\phi_{y}+\frac{\partial \omega_{0}}{\partial y}\right) \\
w(x, y, z)=w_{0}(x, y)
\end{gathered}
$$

$\left(\phi_{x}, \phi_{y}\right)$ denote slopes of a transverse normal at the neutral plane. Reddy's TSDT models are implemented in FGP thermo-mechanical buckling analysis only for rectangular FGP in [27, 35$38,41,44-47,49,54,79,80]$.

\subsubsection{Non-polynomial shear deformation theories (Non-polynomial SDTs)}

This is a category of shear deformation theories, in which the proposed displacement fields are not in polynomial form; instead, they may be in trigonometric, hyperbolic or any other nonpolynomial form. In literature of thermo-mechanical buckling analysis of FGP, the only used non-polynomial SDTs is hyperbolic SDT in [43] for a rectangular FGP.

\subsubsection{Four-variable shear deformation theories (Refined SDTs)}

In plate theories (CPT, FSDT, TSDT, Trigonometric SDT, Hyperbolic SDT) there are five variables to find in order to solve the plate problem. These variables are $u_{0}, v_{0}, w_{0}, \phi_{x}$ and $\phi_{y}$. In order to simplify the problem, number of variables can be reduced to only four variables by splitting $w(x, y, z)$ to two parts $\left(w_{b}, w_{s}\right)$, and express the inplane displacements as functions of these two parts as shown in Eq. (5).

$$
\begin{gathered}
u(x, y, z)=u_{0}(x, y)-z \frac{\partial w_{b}}{\partial x}-f(z) \frac{\partial w_{s}}{\partial x} \\
v(x, y, z)=v_{0}(x, y)-z \frac{\partial w_{b}}{\partial y}-f(z) \frac{\partial w_{s}}{\partial y} \\
w(x, y, z)=w_{b}(x, y)+w_{s}(x, y)
\end{gathered}
$$

$w_{b}, w_{s}$ are the bending and shear components of transverse displacement. Now there are just four variables to find $u_{0}, v_{0}, w_{b}$ and $w_{s}$. This refinement, i.e. variables reduction, can be applied 
to any shear deformation theory. In literature of thermo-mechanical buckling analysis of FGP, only Bateni [42] used the four-variable refined SDT.

\subsection{Von-Karman nonlinearities}

Von Karman nonlinearities are commonly used assumptions that consider simplified nonlinearity by assuming small strain with moderate rotation, aka moderately large deformation assumption [58, 62]. In literature, Von Karman nonlinearities are also called Geometrical nonlinearity or kinematic nonlinearities, and it is commonly considered in thermo-mechanical buckling analysis of FGPs. When Von Karman introduced to CPT it can be referred to as CPT with Von Karman nonlinearities, or Von Karman plate theory.

\section{Properties characterization of FGP}

Properties of FGP at any point can be specified using function models or micromechanics models. Following is a brief on the characterization methods those are used in the literature of FGP thermo-mechanical buckling analysis.

\subsection{Function models}

Microstructure of FGP changes continuously, through at least one dimension, from one composition to another. This change may be designed to have any function. Function models may directly describe properties variation or just the microstructure variation, i.e. volumetric ratios of each component. The common functions used in modelling FGPs are listed below.

\subsubsection{Power function model}

Power function model is the most commonly used function in FGP studies. Functionally graded materials that obey power function rule are called (P-FGM). The power function expresses directly property variation or volume fraction change. If it describes the volume fraction change then a micromechanical model, e.g. Voigt, Mori-Tanaka..., is needed to obtain the distributed properties. Power law expressing volume fraction through FGP may have the form of Eq. (6), presented by Amoushahi [65] considering the coordinate system shown in Fig. 6.

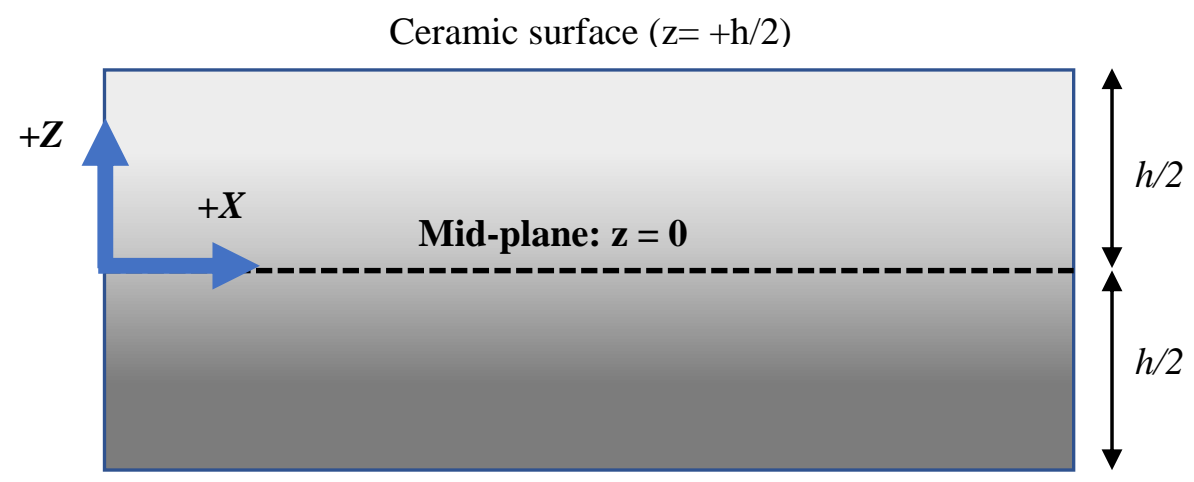

Metal surface $(\mathrm{z}=-\mathrm{h} / 2)$

Fig. 6 FGP's cross section and coordinate system 


$$
v_{c}(z)=\left(\frac{1}{2}+\frac{z}{h}\right)^{n}, \quad \frac{-h}{2} \leq z \leq \frac{h}{2}
$$

$v_{c}(z)$ is the volumetric fraction of ceramic at plane $(z), n$ is the power index value. Note that if the power index $n$ is zero, then the plate will be homogenous plate. If the power index is 1 , then the variation will be linear. At $z=+h / 2$ the material is pure ceramic, $v_{c}(h / 2)=1$. Also at $z=-h / 2$ the material is pure metal $v_{c}(-h / 2)=0$.

\subsubsection{Sigmoid function model}

Functionally graded materials that obey sigmoid power function rule are called (S-FGM) or (SP-FGM). Sigmoid power function is the combination of two different power functions, one for positive range of $z$ and one for the negative range of $z$. Sigmoid power function is used to obtain volume fraction $v_{c}$, then a micromechanical approach, e.g. Voigt, Mori-Tanaka... is used to obtain the distributed properties. Sigmoid power model ensures smooth distribution of the resulting stress $[59,81]$. An example of sigmoid power function is shown in Eq. (7) that is found in [76] considering the coordinate system shown in Fig. 6.

$$
v_{c}(z)= \begin{cases}\left(\frac{1}{2}+\frac{z}{h}\right)^{n}, & \frac{-h}{2} \leq z \leq 0 \\ \left(\frac{1}{2}-\frac{z}{h}\right)^{n}, & 0 \leq z \leq \frac{h}{2}\end{cases}
$$

Sigmoid power function model is rarely used in modeling FGP for thermo-mechanical buckling analysis. Specifically, it has been implemented just by Duc [76] and Chikh [43].

\subsection{Micromechanics models}

Aim of these methods is to predict the effective properties of a composite at given point or lamina as a function of the material and geometric of the composite microstructure using the volumetric ratios obtained by a function model. Daniel [82] categorized these methods as follows.

\subsubsection{Mechanics of materials methods}

These methods are made simple by assuming uniform strain (parallel, rule of mixture, Voigt model) or uniform stress (series, inverse rule of mixture, Reuss model) [82]. Both of them are shown in Eq. (8).

Voigt model (Rule of mixture): $C^{*}=v_{c} C_{c}+v_{m} C_{m}=v_{c} C_{c}+\left(1-v_{c}\right) C_{m}$

$$
\text { Ruess model (Inverse rule of mixture): } C^{*}=\frac{1}{\frac{v_{c}}{C_{c}}+\frac{v_{m}}{C_{m}}}=\frac{1}{\frac{v_{c}}{C_{c}}+\frac{\left(1-v_{c}\right)}{C_{m}}}
$$

$C^{*}$ is the effective property, $C_{c}, C_{m}$ are properties of the consistent, $v_{c}, v_{m}$ are their volume fractions. Voigt model is the most popular in studies of FGP thermo-mechanical buckling analysis. 


\subsubsection{Theory of elasticity methods}

Applies theory of elasticity on a simplified model, resulting in close-form solutions for elastic properties. Common methods are self-consistent methods (effective medium approximation) and Mori-Tanaka method (effective field approximation) [82]. Self-consistent method is used when a crack introduced to the problem [83]. Mori-Tanaka method is used to count for randomness of particulates distribution in a continuous phase [19]. In the literature of thermomechanical buckling analysis of FGPs, Fan [38] used the self-consistent model; and Taj [30] and Sharma $[69,70]$ used Mori-Tanaka model.

\subsubsection{Semi-empirical methods}

Use simple interpolation between bounds obtained by micromechanics methods, while giving comparatively better results [82]. The most common semi-empirical method is Halpin-Tsi method. In the literature of FGP thermo-mechanical buckling analysis, Halpin-Tsai model has been implemented by $\mathrm{Yu}$ [44] and $\mathrm{Wu}[71]$.

\section{Buckling}

Buckling of a plate can be defined as the loss of its stability under compressive loading [84]. That is, the shape of the buckled structure changes into a different configuration when the loads reach some critical value. Buckling occurrence depends on the shape of the structure, properties of the material, loading configuration and boundary conditions. Different bodies buckles in different ways. Columns and flat plates experience bifurcation buckling, aka classical buckling; while curved plates experience snap-through buckling and cylindrical shells experience finite disturbance buckling [62].

\subsection{Stability equation}

Stability equations are the equations derived to obtain buckling loads and their associated mode shapes. Approaches used to obtain stability equations are minimum total potential energy approach, and linear equations approach.

In the first approach, equilibrium equations are derived based on the principle of minimum total potential energy, and then solved for minimum buckling load. Most of studies in literature use this approach in detecting instability.

The second approach is called linear equations approach, which yields stability equations as linear ordinary differential equations, which are easy to solve. This group contains Trefftz criterion and adjacent equilibrium criterion. According to the Trefftz, aka Minimum Potential Energy Difference, criterion, a structure is in a configuration of stable equilibrium if and only if positive change in total potential energy occurs corresponding to any sufficiently small and kinematically admissible displacement. In the literature of FGP thermo-mechanical buckling analysis, Shariyat [29] used Trefftz criterion in detecting instability of perforated FG annular sector plates modeled using 3D elasticity theory. The second approach in the group of linear equation approach is adjacent (neighboring) equilibrium criterion, which considers the equilibrium configuration at the buckling (bifurcation) point and an adjacent configuration that is very close just after buckling point [84]. Many published works used this method, examples can be found at $[39,42,50]$. 


\subsection{FGP buckling}

Aydogdu [85] studied the conditions for bifurcation buckling to exist in the case of FGPs; concluding that for unsymmetrical FGP, under only in-plane compression load, bifurcation can only occur when all edges are clamped. That is because in-plane (stretching) and out-plane (bending) displacements are coupled, just like the case of unsymmetrical laminated plates [62]. Therefore, in-plane load, if even one edge of the unsymmetrical FGP is simply supported the plate will bend. Under uniform shear load bifurcation can occur at plates with clamped as well as simply supported edge, since both of these boundary conditions provide twisting resistance. The stability problem of functionally graded plates is very sensitive to the type of boundary conditions and the form of material variation across the plate thickness. In order for bifurcation point (buckling load) to exist at plate under in-plane compression, initial flatness is necessary. That is, plate has to be initially flat and has to stay flat until buckling occurs. Otherwise, it will not arrive to the bifurcation point, and just exhibits bending deformation. Initial flatness may be interrupted not only by in-plane loads with unclamped boundary conditions, but also by initial imperfections in the structure. Under biaxial in-plane compression, if loads suitably selected, in-plane strain may vanish, then bifurcation point will occur on simply supported plates even if the plate is non-symmetric. Pre-buckling status, i.e. stress and strain, has to studied before conducting buckling studies [62]. An attempt to decouple the stretching and bending deformations, in-plane loads/boundary conditions made to act on the physical neutral plane [86]. Physical neutral plane is the plane that has neither stress nor strain under pure bending. According to Zhang [87] using coordinate system shown in

Fig. 7, the discrepancy between the physical neutral plane and the mid-plane $(C)$ can be calculated using the relation in Eq. (9). In Eq. (9) $E(z)$ is the function that describes the variation of the elasticity module through the thickness of the plate. Note that if the plate is homogeneous, i.e. $E(z)=$ const., $C$ will vanish and the two planes will coincide on each other.

$$
C=\int_{-\frac{h}{2}}^{+\frac{h}{2}} E(z) z d z / \int_{-\frac{h}{2}}^{+\frac{h}{2}} E(z) d z
$$

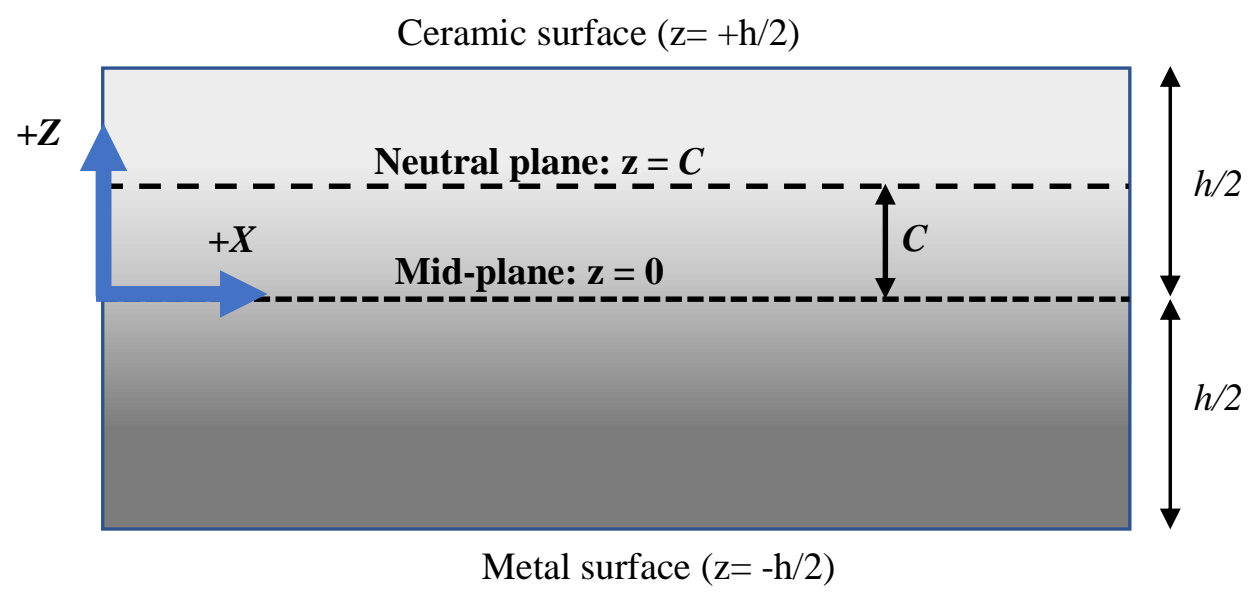

Fig. 7 Neutral and mid planes of FGP 
Initial geometry imperfections have been considered in many published studies. Normally initial imperfections are introduced to the analysis as pre-existing lateral deformation. Recent articles that consider initial imperfections are [25, 37, 79].

\subsection{FGP thermal buckling}

Temperature generates in-plane compression load between every two opposite inextensible edges. If the neutral plane is assumed to be coincident with the mid-plane of non-symmetrical FGP under thermal load, all edges has to be clamped in order to bifurcation point to occur [88]. If all edges are free to expand, i.e. movable, there will be no in-plane thermal stress and the temperature effect will be only on properties of the materials. For symmetrical FGP, if the plate is initially flat, it remains flat until bifurcation point occurs. Initiation of lateral deflection under the applied load, below bifurcation point, results into the plate bending and the bifurcation never occurs, instead of that, it becomes a bending problem. This condition occurs when a rectangular FGP with simply supported boundary conditions is under thermal load, provided that the material distribution across the plate thickness is not symmetric with respect to plate's midplane unless physical neutral plane is considered [62, 85]. Swaminathan [19] presented a comprehensive review on thermal analysis of FGPs.

\subsection{Thermal loads}

Thermal loads applied on a FGP can be expressed by constant or changing temperature through one dimension or more. Temperature variation may be given directly as a spatial function or derived from heat conduction problem. Temperature variation equation, normally through thickness, may be a constant, e.g. [68, 89], linear, e.g. [89, 90] or nonlinear equation, e.g. [34, 80]. Temperature variation may be also given as heat conduction problem as in $[32,33,42,49$, 91].

\subsection{Temperature-dependent FGP}

Thermal load does not just generate stresses in the structure, it also affect the structure's material properties. Considering this effect of temperature rises the complexity level of the analysis. In the literature on FGP thermo-mechanical buckling, temperature-dependency effect on the analysis results has been studied by Shukla [68], Lal [80] and Jari [78] by comparing results of analysis with and without considering martial properties dependency on temperature. Other studies those considered temperature-dependent material properties are [24, 25, 27, 30, $32,35,38,42,45,48,49,53,66,69,70,72,76,77,92]$. Examples of studies those considered temperature-independent material properties are [22, 23, 26, 29, 36, 41, 50, 65].

\subsection{FGP thermo-mechanical buckling}

FGP, as functionally graded materials, are expected to withstand highly elevated temperature without failure. Normally, in addition to the thermal loads there are mechanical loads acting. In that case, the FGP has to withstand both the thermal and mechanical loads at the same time. There is a huge amount of published works deal with mechanical buckling or/and thermal buckling of FGP separately, but small amount of them treat the thermo-mechanical buckling case as defined here as buckling due to simultaneous application of thermal and mechanical loads. This situation is complicated enough so that until now no exact solution available even for the simplest configuration possible. Existence of bifurcation point has to be examined through the analysis of pre-buckling state of the plate. Zhang [92] and Kowal-Michalska [22] attempted to apply the concept of physical neutral plane. 


\section{Solution procedures}

There are many solution methods have been applied in various FGP analysis problems. Solution methods can be categorized as exact, semi analytical and approximate methods. However, only approximate methods have been applied to solve the thermo-mechanical buckling of FGP. Approximate methods contain two main categories, approximately analytical methods and numerical methods. Many methods fall under this category. However only few have been applied to solve the buckling of FGP under combined thermal and mechanical loads.

\subsection{Approximate analytical methods}

These methods seek to obtain approximate solution as a function. This group contains the following methods.

\subsubsection{Ritz method}

Ritz method is a simple and convenient method based on the principle of minimum potential energy. In literature of thermo-mechanical buckling of FGPs it is found that Ritz method has been used in snap-through buckling of thermally post-buckled plate analyses by: Kiani [32], who analyzed static and dynamic case for circular FGPs using Ritz method with Gram-Schmidt process; and Zhang [92], who analyzed the static case of rectangular FGP using multi-term Ritz method.

\subsubsection{Galerkin method}

Galerkin method is a special case of the weighted residual method. This method deals with the governing differential equation directly instead of the energy functional. Therefore, Galerkin method is more general than Ritz method [58]. Galerkin method is one of the most commonly used methods in analyzing thermo-mechanical buckling of FGPs. Duc and various co-authors investigated thermo-mechanical buckling and post-buckling of FGPs based on: CPT [66], FSDT [24, 53] and Reddy's TSDT [26, 41]. Other researchers also presented thermomechanical buckling analysis of symmetric FGPs [43, 76], stiffened FGPs [25, 27, 35], porous FGPs [37], square FGP based on CLPT [22] and rectangular FGP based on four-variable refined plate theory by using multi-term Galerkin method [42].

\subsubsection{Perturbation methods}

Perturbation methods can solve various boundary-value problems in elastic structures[93], by starting from the exact solution of a related, simpler problem. There are main two versions of this method: traditional and two-step perturbation methods. Fallah [67] compared the two variations of this method, i.e. one- and two- step perturbation method, for nonlinear analysis of FGP with thermo-mechanical loading, and concluded that the two-step method must be used instead of the one-step method. Fellah [33] also applied two-step perturbation method to investigate thermo-mechanical buckling of circular FGP. This method is the most used one in analysis of thermo-mechanical buckling of FGPs. Shen and his various co-authors used twostep perturbation method repeatedly to investigate thermo-mechanical buckling and postbuckling of various continuous, laminated and sandwich FG reinforced plates, with or without elastic foundation $[44,46,49,54,79]$ in various thermal environments, and in hygrothermal environment [45]. Fan [38] used two-perturbation method to investigate thermo-mechanical buckling of cracked FG reinforced plate. 


\subsubsection{Power series Frobenius method}

This method aims to find an infinite series solution for ordinary differential equations. Yaghoobi [50] used this method to obtain approximate analytical solution for the ordinary differential equations those have been reduced analytically from the governing equations of stability to investigate thermo-mechanical buckling of FGP.

\subsubsection{Fast converging finite double Chebyshev series}

In this method, displacement functions and loading are approximated in space domain by finite degree double Chebyshev polynomials. This method used by Shukla [68] to investigate thermomechanical post-buckling of FGP.

\subsection{Numerical methods}

Numerical methods obtain approximate solution as a value at many points of the solution domain. These methods are used in order to avoid difficulties associated with the analytical solution methods [56].

\subsubsection{Finite element methods (FEM)}

Finite element method is a powerful method used in solving differential equations those describe engineering systems. The concept behind these methods is that they divide the problem domain into smaller simpler geometric shapes; so they deal with the problem of interest as assemblage of sub-problems those are simpler and easier to solve [20]. FEMs have been used in the analysis of thermo-mechanical buckling of FGPs based on various theories. Following, FEM implementations are categorized based on the considered model of the FGP.

Based on various plate theories (e.g. FSDT, TSDT): Recently, Moita [94] used FEM based on Reddy's TSDT, to compare between linear and nonlinear mechanical and thermo-mechanical buckling of rectangular FGP. FEM has been also used in investigation of thermo-mechanical buckling of: rectangular FGPs by Talha [77], skew FGPs by Taj [30], rectangular FGP with an elliptical cutout by Abolghasemi [73], and sandwich FGPs by Chen [95]. Lal and co-authors investigated stochastic thermo-mechanical buckling and post-buckling of FGPs with holes [36], and further included temperature dependency [80]. Sharma imbedded elasto-plasticity to the thermo-mechanical buckling and post-buckling investigation of FGPs with cutout [70] and further included temperature dependency [69]. Mania [23] investigated the dynamic thermomechanical buckling in addition to the static buckling. Recently, Correia [96] conducted multiobjective optimization study including buckling analysis of thermo-mechanical loading for FGP using FEM.

Based on theory of elasticity: Shariyat [29] investigated the thermo-mechanical buckling of perforated annular sector using curved 3D B-splined C2-continuous element.

Many commercial finite element packages provide the ability to model FGPs based on both plate theories and 3D elasticity theory. Discussion of these packages is out of the scope of this review. However, detailed illustration to model and conduct buckling analysis of rectangular plates including FGPs using models based on FSDT and 3D elasticity theory using ANSYS ${ }^{\circledR}$ APDL [97] is provided by Hassan [98]. 


\subsubsection{Finite strip methods (FSM)}

FSM is a variation of the finite element method, where elements are long strips laid parallel to one another to form the plate [65]. Functionally graded finite strip element is made of mixture of metal and ceramic [99]. This method has many variations depending on the proposed basis shape functions, one of which is the complex finite strip method, which is used by Amoushahi [65] to analysis thermo-mechanical buckling of FGPs.

\subsubsection{Isogeometric analysis (IGA)}

IGA is an integration of spline-based Computer Aided Design (CAD) with finite element method FEM into a single model [78, 100]. IGA bases geometry and solution on same spline function, which makes it easy to have exact description of geometrical complexities [31]. IGA usually based on Non-Uniform Rational B-Spline (NURBS). NURBS-based IGA has been applied by Jari [78] and $\mathrm{Yu}$ [31] in the analysis of the thermo-mechanical buckling of FGPs.

\subsubsection{Differential quadrature method (DQM)}

DQM approximates the partial derivative of a function at a given discrete point as a weighted linear combination of the function values at all the discrete points of the domain [72]. The key to implement DQM is to determine the weighting coefficients [101]. This method is used in investigating the dynamic thermo-mechanical buckling of FGP by Yang [72] and Wu [71]. Mansouri used new DQM that was proposed by Wang [102] to investigate thermo-mechanical buckling of: orthotropic FGPs [103] and orthotropic FGP on elastic foundation in thermal and moisture environment [47].

\subsubsection{Meshless method}

Meshless methods do not require connected nodes. These promising methods continue to improve and get more attention and implementations in engineering field [81, 104, 105]. This category includes wide range of subcategories. Shams [48] used Element-Free Galerkin (EFG) method to investigate thermo-mechanical buckling of FGP.

\subsubsection{Shooting method}

Shooting method solves boundary value problem after converting it to initial value problem, using trial initial conditions and interpolations in order to correct the initial condition until final condition be satisfied [105]. Shooting method has been used in investigating thermomechanical buckling of circular FGP by Li [34].

\section{Concluding remarks and future direction}

The present study presents a review on literature of modelling techniques and solution methods for the thermo-mechanical buckling of functionally graded plates. Following conclusions can be drawn based on the literature reviewed.

There is no publication found to obtain exact elasticity solution or even semi-analytical solution for the FGP buckling under combined thermal and mechanical loads.

Mostly used methods are Galerkin method, Perturbation method and finite element method. Just few solution methods used in this field of analysis. Many other numerical and approximate analytical methods can be implemented and compared with the available results in the literature. 
Rectangular FGPs are the mostly studied; circular FGPs get less attention and skew FGPs are rare in this field. Other general shape of plates are not even exist in this literature.

Stiffened, perforated and cracked FGPs have minimum attention in this field of analysis.

FGP models based on elasticity are rare in this literature. Models based on plate theories are more common.

Relatively new plate models, as Carrera's unified formulation CUF [106], have not implemented yet in this field of study.

Dynamic thermo-mechanical buckling of FGP is a missing topic.

Most of the reported researches include post-buckling study along with the buckling study. Many studies consider the combined thermal and mechanical load only in the post-buckling study, but not in the buckling study. These studies are excluded from this review.

Existing gaps found in the literature of the thermo-mechanical buckling analysis of FGP, offer many chances to contribute. Implementation of the already used solution methods but for other models of FGP, implementation of never used solution methods, obtaining an exact solution for any FGP configuration and implementation of CUF model are suggested topics for future researches.

This review aimed at stimulating researchers to contribute to this field of study, so better analysis tools will be available to designers to make maximum benefit from the promising FGM concept.

\section{References}

[1] Birman, V., Keil, T., Hosder, S., Functionally graded materials in engineering, in Structural Interfaces and Attachments in Biology, Springer New York, 19-41, 2013.

[2] Shen, M., Bever, M., Gradients in polymeric materials. Journal of Materials science, 7(7), 741-746, 1972.

[3] Bever, M., Duwez, P., Gradients in composite materials. Materials Science and Engineering, 10, 18, 1972.

[4] Singh, A.K., Noise Emission form Functionally Graded Materials based Polypropylene Spur GearsA Tribological Investigation. Materials Today: Proceedings, 5(2), 8199-8205, 2018.

[5] Singh, A.K., A novel technique for in-situ manufacturing of functionally graded materials based polymer composite spur gears. Polymer Composites, 40(2), 523-535, 2019.

[6] Tian, X., Zhao, J., Yang, H., Wang, Z., Liu, H., High-speed intermittent turning of GH2132 alloy

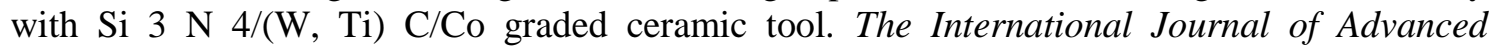
Manufacturing Technology, 100(1-4), 401-408, 2019.

[7] Ngueyep, L.L.M., Ndop, J., Nkene, E.R.A., Ndjaka, J.-M.B., Numerical and Analytical Calculations for Modeling and Designing Drilling Wicks or Rotary Cutters Based of Functionally Graded Materials. Journal of Engineering, 2018, 2018.

[8] Nikbakht, S., Kamarian, S., Shakeri, M., A review on optimization of composite structures Part II: Functionally graded materials. Composite Structures, 2019. 
[9] Hayakawa, N., Oishi, R., Kojima, H., Kato, K., Zebouchi, N. Electric Field Grading by Functionally Graded Materials (FGM) for HVDC Gas Insulated Power Apparatus. IEEE Conference on Electrical Insulation and Dielectric Phenomena (CEIDP), IEEE, 2018.

[10] Radhi, N., Hafiz, M., Atiyah, A., Preparation and Investigation of Corrosion and Biocompatibility Properties for Functionally Graded Materials (NiTi). Ind Eng Manage S, 3, 2169-0316, 2018.

[11] Radhi, N., Preparation And Modeling (Titanium-Hydroxyapatite) Functionally Graded Materials For Bio-Medical Application. International Journal of Civil Engineering and Technology (IJCIET), 9(6), 28-39, 2018.

[12] Dabbagh, A., Madfa, A., Naderi, S., Talaeizadeh, M., Abdullah, H., Abdulmunem, M., Kasim, N.A., Thermomechanical advantages of functionally graded dental posts: A finite element analysis. Mechanics of Advanced Materials and Structures, 1-10, 2017.

[13] Mahmoudi, M., Saidi, A.R., Hashemipour, M.A., Amini, P., The use of functionally graded dental crowns to improve biocompatibility: a finite element analysis. Computer methods in biomechanics and biomedical engineering, 21(2), 161-168, 2018.

[14] Satapathy, P.K., Sahoo, B., Panda, L., Das, S. Finite element analysis of functionally graded bone plate at femur bone fracture site. IOP Conference Series: Materials Science and Engineering, IOP Publishing, 2018.

[15] Han, C., Li, Y., Wang, Q., Wen, S., Wei, Q., Yan, C., Hao, L., Liu, J., Shi, Y., Continuous functionally graded porous titanium scaffolds manufactured by selective laser melting for bone implants. Journal of the mechanical behavior of biomedical materials, 80, 119-127, 2018.

[16] Kumar, G.M. Functionally graded bio-ceramic reinforced PVA hydrogel composites for knee joint artificial cartilages. AIP Conference Proceedings, AIP Publishing, 2018.

[17] Cooley, W.G., Application of functionally graded materials in aircraft structures, in Department of Aeronautics and Astronautics. 2005, Air University.

[18] Swaminathan, K., Naveenkumar, D.T., Zenkour, A.M., Carrera, E., Stress, vibration and buckling analyses of FGM plates: A state of the art review. Composite Structures, 120, 10-31, 2015.

[19] Swaminathan, K., Sangeetha, D.M., Thermal analysis of FGM plates - A critical review of various modeling techniques and solution methods. Composite Structures, 160, 43-60, 2017.

[20] Reddy, J.N., Theory and analysis of elastic plates and shells; CRC press, 2006.

[21] Ventsel, E., Krauthammer, T., Thin plates and shells: theory: analysis, and applications; CRC press, 2001.

[22] Kowal-Michalska, K., Mania, R.J., Static and dynamic thermomechanical buckling loads of functionally graded plates. Mechanics and Mechanical Engineering, 17(1), 99-112, 2013.

[23] Mania, R.J. Dynamic response of FGM thin plate subjected to combined loads. 10th Jubilee Conference on "Shell Structures: Theory and Applications", SSTA, Gdansk, 2013.

[24] Tung, H.V., Thermal and thermomechanical postbuckling of FGM sandwich plates resting on elastic foundations with tangential edge constraints and temperature dependent properties. Composite Structures, 131, 1028-1039, 2015. 
[25] Cong, P.H., Ngoc An, P.T., Duc, N.D., Nonlinear stability of shear deformable eccentrically stiffened functionally graded plates on elastic foundations with temperature-dependent properties. Science and Engineering of Composite Materials, 24(3), 455-469, 2017.

[26] Bakora, A., Tounsi, A., Thermo-mechanical post-buckling behavior of thick functionally graded plates resting on elastic foundations. Structural Engineering and Mechanics, 56(1), 85-106, 2015.

[27] Duc, N.D., Cong, P.H., Quang, V.D., Thermal stability of eccentrically stiffened FGM plate on elastic foundation based on Reddy's third-order shear deformation plate theory. Journal of Thermal Stresses, 39(7), 772-794, 2016.

[28] Aghazadeh, R., Dag, S., Cigeroglu, E., Thermal effect on bending, buckling and free vibration of functionally graded rectangular micro-plates possessing a variable length scale parameter. Microsystem Technologies, 1-24, 2018.

[29] Shariyat, M., Behzad, H., Shaterzadeh, A.R., 3D thermomechanical buckling analysis of perforated annular sector plates with multiaxial material heterogeneities based on curved B-spline elements. Composite Structures, 188, 89-103, 2018.

[30] Taj, M.N.A.G., Chakrabarti, A., Buckling analysis of functionally graded skew plates: An efficient finite element approach. International Journal of Applied Mechanics, 5(4), 2013.

[31] Yu, T., Yin, S., Bui, T.Q., Liu, C., Wattanasakulpong, N., Buckling isogeometric analysis of functionally graded plates under combined thermal and mechanical loads. Composite Structures, $162,54-69,2017$.

[32] Kiani, Y., Axisymmetric static and dynamics snap-through phenomena in a thermally postbuckled temperature-dependent FGM circular plate. International Journal of Non-Linear Mechanics, 89, $1-13,2017$.

[33] Fallah, F., Nosier, A., Nonlinear behavior of functionally graded circular plates with various boundary supports under asymmetric thermo-mechanical loading. Composite Structures, 94(9), 2834-2850, 2012.

[34] Li, S.R., Zhang, J.H., Zhao, Y.G., Nonlinear thermomechanical post-buckling of circular FGM plate with geometric imperfection. Thin-Walled Structures, 45(5), 528-536, 2007.

[35] Van Dung, D., Nga, N.T., Buckling and postbuckling nonlinear analysis of imperfect FGM plates reinforced by FGM stiffeners with temperature-dependent properties based on TSDT. Acta Mechanica, 227(8), 2377-2401, 2016.

[36] Lal, A., Neeranjan Singh, H., Shegokar, N.L., FEM model for stochastic mechanical and thermal postbuckling response of functionally graded material plates applied to panels with circular and square holes having material randomness. International Journal of Mechanical Sciences, 62(1), 18-33, 2012.

[37] Cong, P.H., Chien, T.M., Khoa, N.D., Duc, N.D., Nonlinear thermomechanical buckling and postbuckling response of porous FGM plates using Reddy's HSDT. Aerospace Science and Technology, 77, 419-428, 2018.

[38] Fan, Y., Wang, H., Nonlinear bending and postbuckling analysis of matrix cracked hybrid laminated plates containing carbon nanotube reinforced composite layers in thermal environments. Composites Part B: Engineering, 86, 1-16, 2016.

[39] Mahmoudi, A., Benyoucef, S., Tounsi, A., Benachour, A., Adda Bedia, E.A., Mahmoud, S.R., A refined quasi-3D shear deformation theory for thermo-mechanical behavior of functionally 
graded sandwich plates on elastic foundations. Journal of Sandwich Structures and Materials, 2017.

[40] Shahsavari, D., Shahsavari, M., Li, L., Karami, B., A novel quasi-3D hyperbolic theory for free vibration of FG plates with porosities resting on Winkler/Pasternak/Kerr foundation. Aerospace Science and Technology, 72, 134-149, 2018.

[41] Duc, N.D., Van Tung, H., Mechanical and thermal postbuckling of higher order shear deformable functionally graded plates on elastic foundations. Composite Structures, 93(11), 2874-2881, 2011.

[42] Bateni, M., Kiani, Y., Eslami, M.R., A comprehensive study on stability of FGM plates. International Journal of Mechanical Sciences, 75, 134-144, 2013.

[43] Chikh, A., Bakora, A., Heireche, H., Houari, M.S.A., Tounsi, A., Adda Bedia, E.A., Thermomechanical postbuckling of symmetric S-FGM plates resting on Pasternak elastic foundations using hyperbolic shear deformation theory. Structural Engineering and Mechanics, 57(4), 617639, 2016.

[44] Yu, Y., Shen, H.S., Wang, H., Hui, D., Postbuckling of sandwich plates with graphene-reinforced composite face sheets in thermal environments. Composites Part B: Engineering, 135, 72-83, 2018.

[45] Shen, H.S., Zhang, C.L., Non-linear analysis of functionally graded fiber reinforced composite laminated plates, Part I: Theory and solutions. International Journal of Non-Linear Mechanics, 47(9), 1045-1054, 2012.

[46] Shen, H.S., Zhu, Z.H., Postbuckling of sandwich plates with nanotube-reinforced composite face sheets resting on elastic foundations. European Journal of Mechanics, A/Solids, 35, 10-21, 2012.

[47] Mansouri, M.H., Shariyat, M., Biaxial thermo-mechanical buckling of orthotropic auxetic FGM plates with temperature and moisture dependent material properties on elastic foundations. Composites Part B: Engineering, 83, 88-104, 2015.

[48] Shams, S., Soltani, B., Memar Ardestani, M., The effect of elastic foundations on the buckling behavior of functionally graded carbon nanotube-reinforced composite plates in thermal environments using a meshfree method. Journal of Solid Mechanics, 8(2), 262-279, 2016.

[49] Shen, H.S., Li, S.R., Postbuckling of sandwich plates with FGM face sheets and temperaturedependent properties. Composites Part B: Engineering, 39(2), 332-344, 2008.

[50] Yaghoobi, H., Yaghoobi, P., Buckling analysis of sandwich plates with FGM face sheets resting on elastic foundation with various boundary conditions: An analytical approach. Meccanica, 48(8), 2019-2035, 2013.

[51] Hussein, O.S., Mulani, S.B. Two-dimensional optimization of functionally graded material plates subjected to buckling constraints. 58th AIAA/ASCE/AHS/ASC Structures, Structural Dynamics, and Materials Conference, American Institute of Aeronautics and Astronautics Inc, AIAA, 2017.

[52] Demirbas, M.D., Apalak, M.K., Thermal stress analysis of one- and two-dimensional functionally graded plates subjected to in-plane heat fluxes. Proceedings of the Institution of Mechanical Engineers, Part L: Journal of Materials: Design and Applications, 2017.

[53] Duc, N.D., Tung, H.V., Mechanical and thermal postbuckling of shear-deformable FGM plates with temperature-dependent properties. Mechanics of Composite Materials, 46(5), 461-476, 2010 . 
[54] Shen, H.S., Zhu, Z.H., Buckling and postbuckling behavior of functionally graded nanotubereinforced composite plates in thermal environments. Computers, Materials and Continua, 18(2), 155-182, 2010.

[55] Reddy, J.N., Mechanics of laminated composite plates and shells: theory and analysis; CRC press, 2004.

[56] Szilard, R., Theories and applications of plate analysis: classical, numerical and engineering methods; John Wiley \& Sons, 2004.

[57] Jones, R.M., Buckling of bars, plates, and shells; Bull Ridge Corporation, 2006.

[58] Bhaskar, K., Varadan, T., Plates: theories and applications; John Wiley \& Sons, 2014.

[59] Carrera, E., Fazzolari, F.A., Cinefra, M., Thermal Stress Analysis of Composite Beams, Plates and Shells: Computational Modelling and Applications; Academic Press, 2016.

[60] Chakraverty, S., Pradhan, K.K., Vibration of functionally graded beams and plates; Academic Press, 2016.

[61] Elishakoff, I., Pentaras, D., Gentilini, C., Mechanics of functionally graded material structures; World Scientific, 2016.

[62] Eslami, M.R., Buckling and Postbuckling of Beams, Plates, and Shells, 1; Springer, 2017.

[63] Thai, H.T., Kim, S.E., A review of theories for the modeling and analysis of functionally graded plates and shells. Composite Structures, 128, 70086, 2015.

[64] Abrate, S., Di Sciuva, M., Equivalent single layer theories for composite and sandwich structures: A review. Composite Structures, 179, 482-494, 2017.

[65] Amoushahi, H., Lajevardi, M.M., Buckling of functionally graded plates under thermal, axial, and shear in-plane loading using complex finite strip formulation. Journal of Thermal Stresses, 41(2), 182-203, 2018.

[66] Tung, H.V., Duc, N.D., Nonlinear analysis of stability for functionally graded plates under mechanical and thermal loads. Composite Structures, 92(5), 1184-1191, 2010.

[67] Fallah, F., Nosier, A., Sharifi, M., Ghezelbash, F., On perturbation method in mechanical, thermal and thermo-mechanical loadings of plates: Cylindrical bending of FG plates. ZAMM Zeitschrift fur Angewandte Mathematik und Mechanik, 96(2), 217-232, 2016.

[68] Shukla, K.K., Kumar, K.V.R., Pandey, R., Nath, Y., Postbuckling response of functionally graded rectangular plates subjected to thermo-mechanical loading. International Journal of Structural Stability and Dynamics, 7(3), 519-541, 2007.

[69] Sharma, K., Kumar, D., Elastoplastic stability and failure analysis of FGM plate with temperature dependent material properties under thermomechanical loading. Latin American Journal of Solids and Structures, 14(7), 1361-1386, 2017.

[70] Sharma, K., Kumar, D., Elastoplastic analysis of FGM plate with a central cutout of various shapes under thermomechanical loading. Journal of Thermal Stresses, 40(11), 1417-1441, 2017.

[71] Wu, H., Yang, J., Kitipornchai, S., Parametric instability of thermo-mechanically loaded functionally graded graphene reinforced nanocomposite plates. International Journal of Mechanical Sciences, 135, 431-440, 2018. 
[72] Yang, S.F., Chen, H., Ran, C., Dynamic stability analysis of functionally graded plates subjected to complex loads, in 4th International Conference on Civil Engineering, Architecture and Building Materials, CEABM 2014. 2014, Trans Tech Publications Ltd: Haikou. p. 679-686.

[73] Abolghasemi, S., Shaterzadeh, A.R., Rezaei, R., Thermo-mechanical buckling analysis of functionally graded plates with an elliptic cutout. Aerospace Science and Technology, 39, 250$259,2014$.

[74] Reddy, J., A refined nonlinear theory of plates with transverse shear deformation. International Journal of solids and structures, 20(9-10), 881-896, 1984.

[75] Reddy, J., A general non-linear third-order theory of plates with moderate thickness. International Journal of Non-Linear Mechanics, 25(6), 677-686, 1990.

[76] Duc, N.D., Cong, P.H., Nonlinear postbuckling of symmetric S-FGM plates resting on elastic foundations using higher order shear deformation plate theory in thermal environments. Composite Structures, 100, 566-574, 2013.

[77] Talha, M., Singh, B.N., Thermo-mechanical buckling analysis of finite element modeled functionally graded ceramic-metal plates. International Journal of Applied Mechanics, 3(4), 867880, 2011.

[78] Jari, H., Atri, H.R., Shojaee, S., Nonlinear thermal analysis of functionally graded material plates using a NURBS based isogeometric approach. Composite Structures, 119, 333-345, 2014.

[79] Shen, H.S., Xiang, Y., Lin, F., Hui, D., Buckling and postbuckling of functionally graded graphenereinforced composite laminated plates in thermal environments. Composites Part B: Engineering, 119, 67-78, 2017.

[80] Lal, A., Jagtap, K.R., Singh, B.N., Post buckling response of functionally graded materials plate subjected to mechanical and thermal loadings with random material properties. Applied Mathematical Modelling, 37(5), 2900-2920, 2013.

[81] Sayyad, A.S., Ghugal, Y.M., Modeling and analysis of functionally graded sandwich beams: A review. Mechanics of Advanced Materials and Structures, 1-20, 2018.

[82] Daniel, I.M., Ishai, O., Daniel, I.M., Daniel, I., Engineering mechanics of composite materials, 3; Oxford university press New York, 1994.

[83] Laws, N., Dvorak, G.J., Hejazi, M., Stiffness changes in unidirectional composites caused by crack systems. Mechanics of Materials, 2(2), 123-137, 1983.

[84] Yoo, C.H., Lee, S., Stability of structures: principles and applications; Elsevier, 2011.

[85] Aydogdu, M., Conditions for functionally graded plates to remain flat under in-plane loads by classical plate theory. Composite Structures, 82(1), 155-157, 2008.

[86] Zhang, D.G., Zhou, Y.H., A theoretical analysis of FGM thin plates based on physical neutral surface. Computational Materials Science, 44(2), 716-720, 2008.

[87] Zhang, D.-G., Zhou, Y.-H., A theoretical analysis of FGM thin plates based on physical neutral surface. Computational Materials Science, 44(2), 716-720, 2008.

[88] Kiani, Y., Bagherizadeh, E., Eslami, M.R., Thermal buckling of clamped thin rectangular FGM plates resting on Pasternak elastic foundation (Three approximate analytical solutions). ZAMM - 
Journal of Applied Mathematics and Mechanics / Zeitschrift für Angewandte Mathematik und Mechanik, 91(7), 581-593, 2011.

[89] Na, K.S., Kim, J.H. Three-dimensional thermomechanical buckling of functionally graded plates. 11th International Conference on Fracture, ICF11, Turin, 2005.

[90] Najafizadeh, M.M., Hedayati, B., Refined theory for thermoelastic stability of functionally graded circular plates. Journal of Thermal Stresses, 27(9), 857-880, 2004.

[91] Na, K.S., Kim, J.H. Volume fraction optimization of functionally graded composite plates for stress reduction and thermo-mechanical buckling. 9th International Conference on Multiscale and Functionally Graded Materials, FGM IX, Oahu Island, HI, 2008.

[92] Zhang, D.G., Zhou, H.M., Mechanical and thermal post-buckling analysis of FGM rectangular plates with various supported boundaries resting on nonlinear elastic foundations. Thin-Walled Structures, 89, 142-151, 2015.

[93] Shen, H.-S., A two-step perturbation method in nonlinear analysis of beams, plates and shells; John Wiley \& Sons, 2013.

[94] Moita, J.S., Araújo, A.L., Correia, V.F., Soares, C.M.M., Herskovits, J., Buckling and nonlinear response of functionally graded plates under thermo-mechanical loading. Composite Structures, 2018.

[95] Chen, C.S., Liu, F.H., Chen, W.R., Vibration and stability of initially stressed sandwich plates with FGM face sheets in thermal environments. Steel and Composite Structures, 23(3), 251-261, 2017.

[96] Correia, V.M.F., Madeira, J.F.A., Araújo, A.L., Soares, C.M.M., Multiobjective optimization of functionally graded material plates with thermo-mechanical loading. Composite Structures, 207, 845-857, 2019.

[97] ANSYS® Help System. Mechanical APDL, ANSYS Parametric Design Language Guide. Release 17.1.

[98] Hassan, A.H.A., Kurgan, N., Modeling and Buckling Analysis of Rectangular Plates in ANSYS. International Journal Of Engineering \& Applied Sciences, 11(1), 310-329, 2019.

[99] Ghannadpour, S.A.M., Ovesy, H.R., Nassirnia, M., An investigation on buckling behaviour of functionally graded plates using finite strip method, in 2012 International Conference on Mechanical Engineering and Materials, ICMEM 2012. 2012: Melbourne, VIC. p. 1470-1476.

[100] Lieu, Q.X., Lee, J., Lee, D., Lee, S., Kim, D., Lee, J., Shape and size optimization of functionally graded sandwich plates using isogeometric analysis and adaptive hybrid evolutionary firefly algorithm. Thin-Walled Structures, 124, 588-604, 2018.

[101] Liew, K.M., Huang, Y.Q., Bending and buckling of thick symmetric rectangular laminates using the moving least-squares differential quadrature method. International Journal of Mechanical Sciences, 45(1), 95-114, 2003.

[102] Wang, X., Tan, M., Zhou, Y., Buckling analyses of anisotropic plates and isotropic skew plates by the new version differential quadrature method. Thin-Walled Structures, 41(1), 15-29, 2003.

[103] Mansouri, M.H., Shariyat, M., Thermal buckling predictions of three types of high-order theories for the heterogeneous orthotropic plates, using the new version of DQM. Composite Structures, 113(1), 40-55, 2014. 
[104] Chen, Y., Lee, J., Eskandarian, A., Meshless methods in solid mechanics; Springer Science \& Business Media, 2006.

[105] Fasshauer, G.E., Meshfree approximation methods with MATLAB, 6; World Scientific, 2007.

[106] Carrera, E., Cinefra, M., Petrolo, M., Zappino, E., Finite element analysis of structures through unified formulation; John Wiley \& Sons, 2014. 